\title{
The impact of covid-19 lockdown on maxillofacial related services at
} tertiary dental institution

\author{
SADJ November 2021, Vol. 76 No. 10 p613 - p617 \\ BR Legalamitloa', IT Munzhelele ${ }^{2}$, K Syebele ${ }^{3}$, PD Motloba ${ }^{4}$
}

\begin{abstract}
Background

In the wake of the devastating COVID-19 pandemic, many countries in the world instituted various protocols to limit the spread of the disease and to reduce the burden on health care facilities. However, the unintended consequences of these restrictions included the reduction of human mobility, limited access to health care services, resulting in delayed or missed medical treatment.
\end{abstract}

\section{Aims}

The aim of the study was to assess the impact of the COVID-19 lockdown restriction on maxillofacial services.

\section{Methodology}

This retrospective and descriptive study of patient's clinical records, spanned from October 2019 to August 2020.This period included the pre-lockdown (October -December 2019) and the different lockdown (levels 5,4, and 3) periods. Complete clinical patient records were included for analysis.

\section{Author affiliations:}

1. Batlhalefi R Legalamitloa: Bachelor of Dental Surgery (University of Limpopo). Department of Oral and maxillofacial Surgery, School of Oral Health Sciences, Sefako Makgatho Health Sciences University, Pretoria, South Africa. ORCID Number: 0000-0003-4922-3822

2. Thifhelimbilu Munzhelele: Bachelor of Dental Surgery (Medunsa), MBChB(UP), MChD-Max-Fax MED (UP), FCMFOS (SA), Dip Odont (UP), PGDHSE (WITS), Department of Oral and maxillofacial Surgery, School of Oral Health Sciences, Sefako Makgatho Health Sciences University, Pretoria, South Africa, ORCID Number: 00000002-0082-9391

3. Kabunda Syebele: Med.Dip, Dental Dip, OMFSurg Dip (Kinshasa), Dip.Odont(Oral Surg) (Pretoria), MSc. Odont (Oral Surgery) (Pretoria). Department of Oral and maxillofacial Surgery, School of Oral Health Sciences, Sefako Makgatho Health Sciences University, Pretoria, South Africa, ORCID Number: 0000-0003-0960-0822

4. Pagollang D Motloba: BDS (Medunsa), MPH (Epidemiology) (Tulane), Dent (Comm Dent) Head, Department of Community Dentistry, School of Oral Health Sciences, Sefako Makgatho Health Sciences University. ORCID Number: 0000-0003-1379-7576

\section{Corresponding author:}

Batlhalefi R Legalamitloa: Department of Oral and Maxillofacial Surgery,

School of Oral Health Sciences, Sefako Makgatho Health Sciences

University, Pretoria, South Africa. Email: blegalamitloa@gmail.com

\section{Author contributions:}

1. BR Legalamitloa: $40 \%$ - Conceptualization, writing, edition and final review

2. IT Munzhelele: $15 \%$ - Conceptualization, edition and final review

3. K. Syebele: $15 \%$ - Conceptualization, edition and final review

4. PD Motloba: $30 \%$ - Data analysis, writing, edition and final review

\section{Results}

The study revealed a $88 \%, 86 \%$, and $45 \%$ decline in maxillofacial and oral surgery consultations during levels 5,4 and 3 respectively. Third molar impaction related complaints such as pericoronitis, pain and sepsis were the predominant consultation motives during all levels of lockdown periods. Gender and age of patients had no impact on the consultation rates during the course of the study.

\section{Conclusion}

The Covid-19 pandemic related lockdown restrictions affected the service provision for maxillofacial patients seen at this institution.

\section{Key Words}

Covid-19, SARS-CoV-2, pandemic, maxillofacial services,

\section{INTRODUCTION}

The COVID-19 pandemic-induced mortality and morbidity affected several millions globally since the outbreak of the disease in Wuhan China in December 2019. ${ }^{1}$ Despite many localized interventions, the global effort has been lackluster and the pandemic continued to wreak havoc especially in under-resourced settings. Tracking and tracing of the infected and affected persons were among the earlystage activities to mitigate the spread of COVID-19. ${ }^{2}$ Travel restrictions were imposed locally and internationally ${ }^{3}$ together with non-pharmaceutical interventions. ${ }^{4}$ To date, social distancing, wearing of masks, and handwashing remains the most cost-effective interventions against COVID-19. ${ }^{5}$ These interventions are aimed at slowing down the spread of the SARS-CoV-2 virus, flattening of the infection curve and therefore limiting the pandemic severity and duration. ${ }^{6}$ The rationale for restricting personal freedom of movement was found in an attempt to protect the capacity and integrity of the health systems infrastructure and resources.

The South African lockdown has 5 alert levels, from the highly restrictive level 5 (hard or total) lockdown to level 1 characterised by the return to some form of normality with limited precautions. ${ }^{7}$ Despite the legislation that permits continuation of essential health services, the pandemic resulted in an unintended decrease in service offerings and a decrease in patient-access to health care services and facilities. The treatment of urgent and emergency services took precedence as the restriction tightened and the pandemic worsened. It was observed that during periods of restrictions, such as pandemics or national 
disaster, healthcare utilization decreases. Elective and preventative services were postponed or delayed, and often patients reported reduced healthcare visits due to fears of contracting the virus in health facilities. ${ }^{8}$

The impact of Covid-19 on the health system varied markedly depending on the type of healthcare facility, nature of services provided, location and size. ${ }^{9}$ The utilization of health services involves a complex interaction between means to access health services (economic and social) and perceptions of the severity of one's condition, and availability of staff and other needs. ${ }^{10}$ The lockdown restrictions influenced utilization of services, depending on the availability of means (transport and finances) and location of the health care facilities.

Globally, dental services were limited to emergency treatment; however, urgent maxillofacial services such as, pain relief, trauma, infection and pathology continued to be provided. ${ }^{11}$ Similarly, facilities providing emergency medical/ dental care and urgent maxillofacial services remained operational during lockdown.

We hypothesised that the successive levels of COVID-19 related lockdown restrictions had an impact on maxillofacial and oral surgery services.

\section{AIM OF THE STUDY}

The aim of the study was to assess the impact of the lockdown restrictions on maxillofacial service provision. To achieve this, two related questions were examined:

(i) would the volume of consultations for maxillofacial and oral surgery services be affected by the Covid-19 pandemic lockdown restrictions? We hypothesized that the outbreak resulted in meaningful reductions in the volume of patients seeking consultation.

(ii) did changes in healthcare services utilization due to the SARS-CoV-2 outbreak differ by gender, age, and time? We hypothesized that these variables influenced patient behaviour regarding the utilization of services.

\section{METHODOLOGY}

Study design

This was a retrospective, records-based descriptive crosssectional study.

Time and setting of the study

The period of the study was from October 2019 to August 2020. It included the sequential phases: normal preCOVID-19 pandemic, and the subsequent different levels of the lockdown periods during the COVID-19 pandemic. The research was based on the audit of complete clinical records of patients that presented to the Department of Maxillofacial and Oral Surgery (MFOS) during the study period. Lockdown periods were categorised as follows (Figure 1):

1. State of Normality - From 1 October to 31 December 2019. This period of quiescence reflected a state of normalcy, and for this study was be used as a reference point for all the changes in service delivery.

2. Pre-lockdown - from 1 January to 31 March 2020, as the world was already sensitised to the impeding pandemic.
Figure 1: South Africa -lockdown alert levels.

\begin{tabular}{|c|c|c|c|c|}
\hline ALERT & ALERT & ALERT & ALERT & ALERT \\
\hline LEVEL & LEVEL & LEVEL & LEVEL & LEVEL \\
\hline $\begin{array}{c}\text { Lrastic measures } \\
\text { to contain the } \\
\text { spread of the } \\
\text { virus and } \\
\text { save lives. }\end{array}$ & $\begin{array}{c}\text { Extreme } \\
\text { precautions to } \\
\text { limit community } \\
\text { transmission } \\
\text { and outbreaks. } \\
\text { while allowing } \\
\text { some activity to } \\
\text { resume. }\end{array}$ & $\begin{array}{c}\text { Restrictions on } \\
\text { many activities. } \\
\text { including at } \\
\text { workplaces } \\
\text { and socially. } \\
\text { to address a } \\
\text { high risk of } \\
\text { transmission. }\end{array}$ & $\begin{array}{c}\text { Physical } \\
\text { distancing } \\
\text { and restrictions } \\
\text { on leisure and } \\
\text { social activitios to } \\
\text { prevent } \\
\text { a resurgence of } \\
\text { the virus. }\end{array}$ & $\begin{array}{c}\text { Most normal } \\
\text { activity can } \\
\text { resume, with } \\
\text { precautions and } \\
\text { health guidelines } \\
\text { followed at all } \\
\text { times. } \\
\text { Population } \\
\text { prepared for } \\
\text { an increase in } \\
\text { alert levels if } \\
\text { necessary. }\end{array}$ \\
\hline
\end{tabular}

3. Level 5 Lockdown: from the 1st to 30 April 2020, hard lockdown.

4. Level 4 lockdown: from the 1st to 31 May 2020, modified hard lockdown.

5. Level 3 Lockdown: from 1 June to August 2020, eased lockdown.

\section{Data sources and outcomes}

We extracted all available data from the health services utilization information system. The records included clinical and demographic data from patients who consulted MFOS section. The accuracy and completeness of the electronic data was validated by comparing representative sample of these records to the physical clinical records. Incomplete clinical records were excluded from the research.

\section{Ethical approval or the study}

The research received ethical approval from the Sefako Makgatho Health Sciences University Research Ethics committee (SMUREC/D/25/2021: PG).

\section{Statistical analysis}

SPSS version 25 was used for all analyses, and the level of significance was set at $\alpha=0.05$. Chi-square tests, Analysis of variance (ANOVA) and Kruskal-Wallis tests evaluated association of variables over different levels of the lockdown.

Figure 2: Consultation by treatment type and time

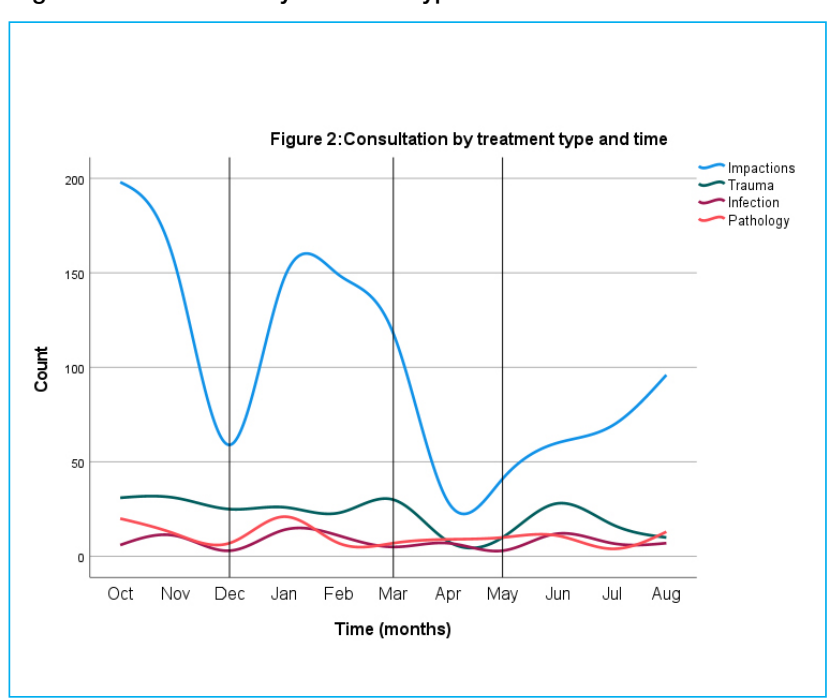




\begin{tabular}{|c|c|c|c|c|c|c|}
\hline & & PERIOD & & & & \\
\hline Variable & Pre Lockdown & Level 5 & Level 4 & Level 3 & Test & $p$-value \\
\hline Visits n (\%) & 899 (55.6) & $105(6.5)$ & $120(7.4)$ & 494(30.5) & & \\
\hline \multicolumn{7}{|l|}{ Gender } \\
\hline Male & $477(53.7)$ & $61(58.1)$ & $72(60.0)$ & $251(50.8)$ & $X^{2}$ & 1 \\
\hline Female & $422(46.9)$ & $44(41.9)$ & $48(40.0)$ & $243(49.2)$ & & 0.223 \\
\hline \multicolumn{7}{|l|}{ Age } \\
\hline Mean(SD) & 32.66(13.73) & 33.96(17.99) & $34.16(16.66)$ & $33.51(18.43)$ & ANOVA & 0.540 \\
\hline Median(IQR) & $30.0(24.0,38.0)$ & $32(23.0,45.0)$ & $30.0(24 \cdot 0,41.75)$ & $31(24.0,39.0)$ & K-W & 0.669 \\
\hline \multicolumn{5}{|l|}{ Consultations } & $x^{2}$ & \\
\hline Impactions & $414(58.4)$ & $29(4.1)$ & $41(5.8)$ & 225(31.7) & & 0.000 \\
\hline Infection & $30(45.5)$ & $7(10.6)$ & $3(4.5)$ & $26(39.4)$ & & 0.133 \\
\hline Pathology & $35(42.7)$ & $9(11.0)$ & $10(12.2)$ & $28(34.1)$ & & 0.40 \\
\hline Trauma & 79(52.0) & $8(5.3)$ & $10(6.6)$ & $55(36.2)$ & & 0.442 \\
\hline \multicolumn{5}{|l|}{ Reviews } & $x^{2}$ & \\
\hline Infection & $82(44.6)$ & $10(5.4)$ & 23(12.5) & $69(37.5)$ & & 0.001 \\
\hline Pathology & $120(65.9)$ & $15(8.2)$ & $13(7.1)$ & $34(18.7)$ & & 0.002 \\
\hline Trauma & $108(56.5)$ & $16(8.4)$ & 19(9.9) & $48(25.1)$ & & 0.160 \\
\hline
\end{tabular}

\section{RESULTS}

The study shows that 1618 patients sought maxillofacial care from 01 January 2020 until 30 August 2020. The utilization of these oral health services declined drastically during the lockdown. The number of patients receiving services at MFOS-SMU was 899 in the pre-lockdown period, 105, 120 and 494 in the lockdown level 5, 4 and 3 periods respectively.

This translates to $88 \%, 86 \%$ and $45 \%$ decline in patient numbers compared to the pre-lockdown levels. Slightly more males than females consulted the institution during all levels of lockdown $(p=0.22)$. The patient-age mean of 33.1, and median 30.0 years, showed no significant differences ( $p=0.54$ and $p=0.67$ ) respectively across the different levels of lockdown 2020.

\section{Figure 3: Reviews by treatment type and time}

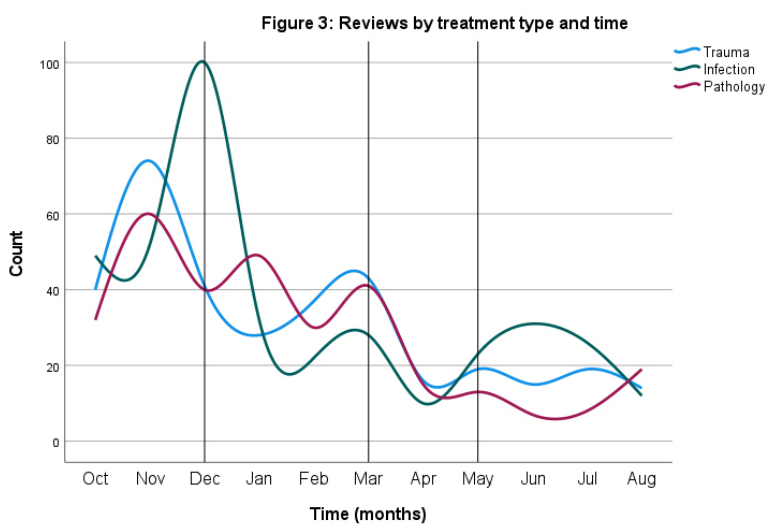

The number of patients who consulted due to impacted third molar (3Ms) problems was higher than all other types of consults during all levels lockdown. Compared to global pre-covid period (October to December 2019), the number of consultations showed a substantial decline. (Figure 2). Consultations for infection, trauma and pathology were more frequent during pre-lockdown and level 3 lockdown. However, these differences were not significant (Table 1). The number of patients reviewed for trauma, pathology or infections were higher than cases of consultations for all levels of the lockdown. Reviewed cases were significantly lower during lockdown levels 5 and 4 , for infection and pathology ( $p=0.001$ and 0.002). Figure 3 shows the marked decline in the number of review cases from October 2019 until the end of May 2021.

Overall, the period of hard lockdown (levels 5 and 4) resulted in a drastic drop in the number of patients reporting at the facility for maxillofacial treatment.

\section{DISCUSSION}

This retrospective study sought to evaluate the impact of the COVID-19 pandemic lockdown restrictions on the utilization of maxillofacial services in a tertiary referral hospital. The study focussed on comparing the volume (quantity) of consultations and the type of clinical findings (quality) reported at the department during the studied periods. This study provides evidence that the pandemic has affected all dental services including urgent and emergency care. The reduction in the number of patients attending maxillofacial services in our setting is immense compared to similar institutions. Our findings show a decline of $88 \%$ and $86 \%$ during level 5 and 4 lockdown periods. In comparison Bartella ${ }^{12}$, Donohoe ${ }^{13}$ and Vishwakarma ${ }^{14}$ reported a $45 \%, 46 \%$ and $73.90 \%$ drop in maxillofacial patients due to COVID-19. We reported similar differences for trauma $9.3 \%$ versus $35 \% .{ }^{13}$; and 
consultations for infection $4.08 \%$ compared to $28 \% .^{13}$ More reviews were recorded than consultations for cases of trauma, infections, and pathology for all periods of the lockdown. Globally, comparatively more cases of maxillofacial trauma, infection and pathology were treated than in our clinic. ${ }^{15,16}$

Most hospitals in the developing world adopted COVID-19 prevention protocols more readily and were thus able to resume maxillofacial operation on a regular basis. The few consultations at this tertiary hospital are indicative of the low number of patients referred from feeder clinics and hospitals. The majority of these institutions reduced their operating times or suspended services altogether during the lockdowns. The change in services times might account for the reduction in referrals for oral pathologies. Patients in the resource constrained environment often face financial, logistical and transport challenges that often derail their access to maxillofacial treatment. The situation worsened during the lockdown as resources were redirected towards the pandemic control. The majority of patients seen in our facility are indigent and rely on state-funded transportation for their hospital access.

Treatment of impacted third molars were performed more frequently during all phases of the lockdown than other services. Impacted third molars are associated with severe pain, pericoronitis, swelling, trismus and other signs of spreading infection. ${ }^{17}$ Therefore, patients suffering from these dental complications are likely to seek help than patients with innocuous soft tissue pathology. Several studies confirm that during the pandemic dental extractions were performed most frequently. ${ }^{18}$

We attribute the decline in the utilization of maxillofacial services in our facility to several reasons: Firstly, the hard lockdown severely restricted the movement of people including visits to healthcare facilities. Secondly, during this period of total shutdown, the world was overwhelmed by anxiety and fear of the virus. Consequently, the majority of people requiring treatment postponed healthcare services, including maxillofacial treatment. ${ }^{15,19}$ Patients were genuinely afraid of being exposed to the virus in hospitals. In their study, Wong and colleagues reported that patients viewed hospitals as infectious reservoirs, "crawling with COVID-19". "The cumulative fear of dentists, of COVID-19 and implementation of travel restrictions provide powerful and justifiable excuses to postpone healthcare services. ${ }^{20}$

Thirdly, the initial shortages in covid tests and the long waiting periods for laboratory results caused unnecessary delays and postponement of urgent maxillofacial treatment. Fourthly, shortages of personal protective equipment (PPEs), critical equipment and consumables had a negative effect on patient treatment and outcomes. During the early phases of the pandemic, the global shortages in PPEs, ventilators and other related equipment had reached catastrophic levels. Despite, instituted rationing, the level of scarcity had an impact on the medical and surgical services. ${ }^{21}$ Lastly, delays in the adoption of COVID-19 protocols in our facility contributed to deferrals and postponement of patient treatment. It is only after these constraints were addressed that some form of normalcy was attained and the patient number started to rise. Still, the maxillofacial services have not returned to pre-covid figures.

\section{CONCLUSION}

The significant decline in the number of patients treated at our facility highlighted the negative impact of the COVID-19 pandemic on maxillofacial service. Healthcare services are vulnerable to pandemics. Therefore, referral systems and infrastructure must be strengthened to support and maintain patient care beyond tertiary centres.

\section{References}

1. Kang S-J, Jung SI. Age-related morbidity and mortality among patients with COVID-19. Infect Chemother. 2020;52(2):154-164.

2. Roderick P, Macfarlane A, Pollock AM. Getting back on track: control of covid-19 outbreaks in the community. BMJ. 2020; 369:m2484. https://doi.org/10.1136/bmj. m2484

3. Chinazzi M, Davis JT, Ajelli M, et al. The effect of travel restrictions on the spread of the 2019 novel coronavirus (COVID-19) outbreak. Science. 2020;368(6489):395400.

4. Bo $\mathrm{Y}$, Guo C, Lin C, et al. Effectiveness of nonpharmaceutical interventions on COVID-19 transmission in 190 countries from 23 January to 13 April 2020. Int J Tuberc Lung Dis 2021;102:247-253.

5. Leung C, Cheng K, Lam T, Migliori G. Mask wearing to complement social distancing and save lives during COVID-19. Int J Tuberc Lung Dis. 2020;24(6):556-558.

6. Lee K-B, Han S, Jeong Y. COVID-19, flattening the curve, and Benford's law. Physica A. 2020;559:125090. https://doi.org/10.1016/j.physa.2020.125090.

7. Hamzelou J.World in lockdown. New Sci. 2020;245(3275):7.https://doi.org/10.1016/S02624079(20)30611-4

8. WongLaura E, HawkinsJessica E, MurrellKaren L. Where are all the patients? Addressing Covid-19 fear to encourage sick patients to seek emergency care. NEJM Catalyst Innovations in Care Delivery. 2020. https://doi. org/10.1056/CAT.20.0193

9. Hatefi S, Smith F, Abou-El-Hossein K, Alizargar J. COVID-19 in South Africa: lockdown strategy and its effects on public health and other contagious diseases. Public Health. 2020;185:159-160.

10. Babitsch B, Gohl D, von Lengerke T. Re-revisiting Andersen's Behavioral Model of Health Services Use: a systematic review of studies from 1998-2011. Psychosoc Med. 2012;9:Doc11-Doc11. https://dx.doi. org/10.3205\%2Fpsm000089

11. Pabst A, Zeller AN, Sader R, et al. The influence of the SARS-CoV-2 pandemic on oral and maxillofacial surgery: a nationwide survey among 54 hospitals and 240 private practices in Germany Clin. Oral Investig... 2021;25(6):3853-3860.

12. Bartella AK, Halama D, Kamal M, et al. Impact of COVID-19 on Oral and Maxillofacial Surgery: Preliminary Results After the Curfew. J. Craniofac. Surg.. 2021;32(3):e305-e308.

13. Donohoe E, Courtney R, McManus E, Cheng J, Barry T. The impact of COVID-19 on Oral and Maxillofacial Surgery patient presentations to the Emergency Department: A West of Ireland Experience. Advances in 
Oral and Maxillofacial Surgery. 2021;2:100061. https:// dx.doi.org/10.1016\%2Fj.adoms.2021.100061

14. Vishwakarma K, Khakhla DH, Ambereen A, Rawat SK, Mishra P, Shukla B. Impact of COVID-19 on oral and maxillofacial surgery practice in India: A national survey. Natl J Maxillofac Surg. 2021;12(2):219-226.

15. Hartnett KP, Kite-Powell A, DeVies J, et al. Impact of the COVID-19 pandemic on emergency department visitsUnited States, January 1, 2019-May 30, 2020. Morbidity and Mortality Weekly Report. 2020;69(23):699-704.

16. $\mathrm{Pu} J \mathrm{JJ}$, McGrath $\mathrm{CP}$, Leung $Y Y$, et al. The Impact of Coronavirus Disease 2019 on the Disease Pattern of Oral and Maxillofacial Surgery Inpatients: A Comparative Study. Front.Med. 2021;8(461). https://doi.org/10.3389/ fmed.2021.613663

17. Renton $\mathrm{T}$, Wilson $\mathrm{NH}$. Problems with erupting wisdom teeth: signs, symptoms, and management. $\mathrm{Br} J$ Gen Pract. 2016;66(649):e606-e608. https://dx.doi. org/10.3399\%2Fbjgp16X686509

18. Fux-Noy A, Mattar L, Shmueli A, Halperson E, Ram D, Moskovitz M. Oral Health Care Delivery for Children During COVID-19 Pandemic-A Retrospective Study. Front. Public Health. 2021;9(504). https://doi.org/10.3389/ fpubh.2021.637351.

19. Jeffery MM, D'Onofrio $G$, Paek $H$, et al. Trends in emergency department visits and hospital admissions in health care systems in 5 states in the first months of the COVID-19 pandemic in the US. JAMA Intern. Med. 2020;180(10):1328-1333.
20. Armfield JM. What goes around comes around: revisiting the hypothesized vicious cycle of dental fear and avoidance. Community Dent. Oral Epidemiol. 2013;41(3):279-287.

21. Jessop ZM, Dobbs TD, Ali SR, et al. Personal protective equipment for surgeons during COVID-19 pandemic: systematic review of availability, usage and rationing. Br J Surg.. 2020;107(10):1262-1280.

\section{YOU ARE ONE PHONE CALL AWAY FROM A SOLUTION!}

\section{SPEOHAL OFFER. (OOT 21 TOMARRH 22)}

\section{LIIUS DEAL WITH YOURE}

\section{*Importing and Exporting Anguish!}

- Procurement of goods for business use

- Freight Forwarding (By air and road)/Logistics

- Customs Clearing

Look no more - we offer best services for all your medical and dental consumables and equipment
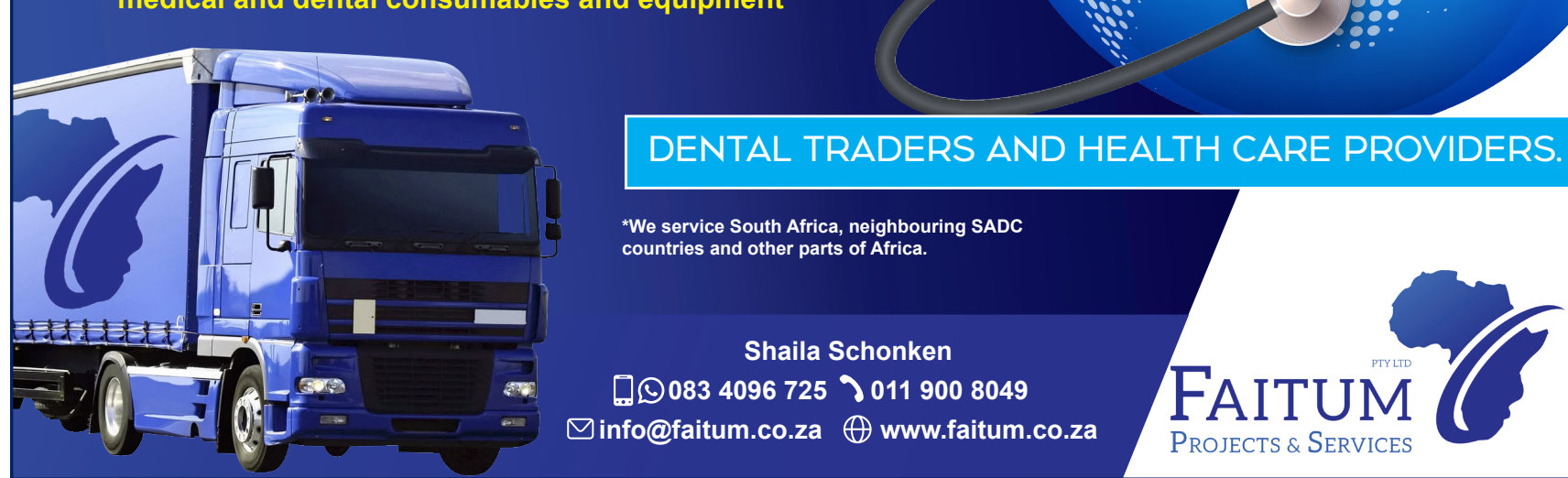

DENTAL TRADERS AND HEALTH CARE PROVIDERS.

*We service South Africa, neighbouring SADC countries and other parts of Africa.

Shaila Schonken

대0834096725 0119008049 ๑info@faitum.co.za $\bigoplus$ www.faitum.co.za

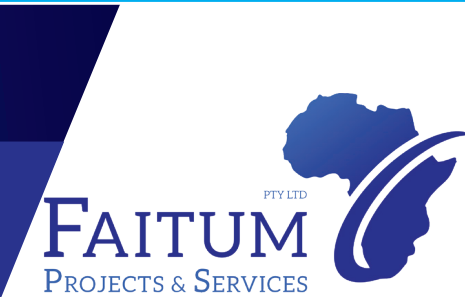

